\title{
DISTRIBUIÇÃO DE METAIS E CARACTERIZAÇÃO DAS CONSTANTES DE TROCA ENTRE ESPÉCIES METÁLICAS E FRAÇÕES HÚMICAS AQUÁTICAS DE DIFERENTES TAMANHOS MOLECULARES
}

\author{
Adriana Barbosa Araújo, André Henrique Rosa e Julio Cesar Rocha* \\ Instituto de Química, Universidade Estadual Paulista, CP 355, 14801-970 Araraquara - SP \\ Luciane Pimenta Cruz Romão \\ Universidade Federal do Sergipe, Jardim Rosa Elze s/n, 49100-000 São Cristóvão - SE
}

Recebido em 30/10/01; aceito em 11/3/02

\begin{abstract}
METAL DISTRIBUTION AND CHARACTERIZATION OF EXCHANGE CONSTANTS BETWEEN METAL SPECIES AND AQUATIC HUMIC FRACTIONS WITH DIFFERENT MOLECULAR SIZES. In this work the metal distribution and exchange constants between metal species and aquatic humic fractions with different molecular sizes were studied. The aquatic humic substances (AHS) were extracted by XAD-8 resin from water sample collected from Itapitanguí river, São Paulo State, Brazil. The AHS were fractionated in six fractions with different molecular sizes $(>100-<5 \mathrm{kDa})$ and characterized by several techniques. Molar ratios H/C suggested higher aromaticity for fractions $\mathrm{F}_{1}$ and $\mathrm{F}_{6}$ whereas molar ratios $\mathrm{C} / \mathrm{N}$ didn't show any differences regarding the humification degree between the fractions. The UV-Vis absorbance $\mathrm{a}_{254} / \mathrm{a}_{436}$ ratio showed higher results for $\mathrm{F}_{4}$ and $\mathrm{F}_{5}$, probably by less condensed features. FTIR studies showed high similarity in the functional groups in the fractions. The highest percentage of traces of $\mathrm{Co}, \mathrm{Al}, \mathrm{Fe}, \mathrm{Mn}, \mathrm{Cu}, \mathrm{Zn}$ and $\mathrm{Ni}$ (determined by ICP-AES) was preferably complexed by fractions $\mathrm{F}_{3}$ and $\mathrm{F}_{4}$ with a greater amount of dissolved organic carbon (DOC). In addition, the exchange constants, determined by ultrafiltration method, showed complexes AHS-Fe and AHS-Al with higher stability than complexes AHS-Co in all fractions.
\end{abstract}

Keywords: aquatic humic substances; metal complexes; exchange constants.

\section{INTRODUÇÃO}

A maior parte do carbono orgânico refratário existente em ambientes aquáticos está na forma de Substâncias Húmicas (SH). Estas são formadas durante a decomposição microbiológica da biomassa ${ }^{1,2} \mathrm{em}$ águas e em solos. As SH são misturas heterogêneas de polieletrólitos, com composição, estrutura, funcionalidades e massas moleculares variadas $^{1,3}$. Diferem de outras classes de compostos naturais devido à sua estrutura química indefinida e gênese complexa $a^{4,5}$. O alto teor de grupos funcionais contendo oxigênio na forma de carboxilas, hidroxilas fenólicas e carbonilas ${ }^{6}$ conferem às $\mathrm{SH}$ papel importante na reatividade e transporte de espécies orgânicas e inorgânicas ${ }^{3,7}$.

A alta capacidade complexante das substâncias húmicas aquáticas (SHA) pode alterar a biodisponibilidade e os efeitos toxicológicos de metais em sistemas aquáticos ${ }^{8-10}$. Os complexos formados entre SHA e espécies metálicas (SHA-M) possuem diferentes estabilidades, as quais são altamente dependentes do $\mathrm{pH}$, espécies metálicas, força iônica, concentração de SHA e condições redox ${ }^{11}$. Recentes investigações concluíram que, para caracterizar a "reatividade" da ligação entre espécies metálicas e SHA, são necessários procedimentos analíticos adequados que forneçam informações sobre a estabilidade termodinâmica e cinética ${ }^{12,13}$. Estudos de troca-iônica têm demonstrado que a disponibilidade de metais ligados às SHA parece ser dependente também de fatores cinéticos ${ }^{14}$. Também, a força e natureza da ligação entre SHA e metais são fortemente influenciadas pelo tamanho molecular das $\mathrm{SHA}^{15}$, cujos pesos moleculares podem variar por exemplo, de $>100 \mathrm{a}<5 \mathrm{kDa}^{11,15}$. Rocha et al. ${ }^{16}$ têm utilizado procedimentos de fracionamento por tamanhos moleculares, para reduzir a polidiversidade das SHA e permitir a caracterização de suas interações com espécies metálicas.

*e-mail: jrocha@iq.unesp.br
Diferentes modelos de complexação e métodos de cálculos também têm sido aplicados na investigação da complexação de metais pelas SHA. A adição de espécies complexantes (p. exemplo Cu, EDTA, DTPA) e aplicação de novas metodologias permitem a investigação da complexação de metais pelas SHA, devido à alteração no equilíbrio entre SHA e espécies metálicas originalmente complexadas. A caracterização da constante de estabilidade entre espécies metálicas com as frações húmicas de diferentes tamanhos moleculares é uma nova contribuição para o entendimento das interações entre metais e matéria orgânica aquática. Desta forma, neste trabalho determinaram-se as constantes de troca entre íons $\mathrm{Cu}$ (II) adicionados e espécies metálicas complexadas nas frações húmicas, utilizando-se procedimento analítico baseado em ultrafiltração (UF). Para tal, fez-se o fracionamento com base no tamanho molecular de SHA extraídas de amostras de água coletadas no Rio Itapanhaú no município de Bertioga-SP. As frações húmicas foram caracterizadas utilizando-se análise elementar, espectrofotometria na região do UV-Vis e do infravermelho. As determinações de carbono orgânico dissolvido (COD) e metais foram feitas utilizando-se método de combustão catalítica e espectrofotometria de emissão atômica, respectivamente. A determinação das constantes de troca foi feita baseada na lei de ação das massas e no procedimento analítico proposto por Burba et al. ${ }^{13}$.

\section{PARTE EXPERIMENTAL}

\section{Reagentes e soluções}

Todos os reagentes utilizados foram de grau e pureza analítica e as soluções preparadas com água desionizada (sistema Milli-Q, Millipore). As soluções ácidas e alcalinas necessárias para a extração das SHA foram preparadas com $\mathrm{HCl} 30 \%$ (Merck AG, previamente purificado por destilação) e $\mathrm{NaOH}$ (suprapur, Merck AG). A resina adsorvente XAD 8 (Serva Feinbiochemica), necessária para a 
extração das SHA, foi previamente purificada por bateladas sucessivas com soluções $\mathrm{HCl} 0,5 \mathrm{~mol} \mathrm{~L}^{-1}, \mathrm{NaOH} 0,5 \mathrm{~mol} \mathrm{~L}^{-1}$ e metanol (duração 24 h cada batelada). Solução padrão diluída de íons $\mathrm{Cu}$ (II) foi preparada a partir de solução estoque de íons $\mathrm{Cu}$ (II) $1000 \mathrm{mg} \mathrm{L}^{-1}$ em $1 \%$ (v/v) de $\mathrm{HNO}_{3}$ (Normex, Carlo Erba).

\section{Extração, purificação e liofilização das substâncias húmicas} aquáticas

Coletaram-se amostras de água em um afluente do Rio Itapanhaú, pertencente ao Parque estadual da Serra do Mar, localizado no $11^{\circ} \mathrm{Gru}$ po de Unidades de Gerenciamento de Recursos Hídricos (UGRHIs), sétima UGRHI-Baixada Santista, município de Bertioga-SP. Após filtração através de membrana $0,45 \mu \mathrm{m}$, fez-se a extração das SHA utilizando-se procedimento recomendado pela "International Humic Substances Society (IHSS)" ${ }^{\prime 17}$. As SHA foram purificadas por diálise para remoção do excesso de sais dissolvidos, segundo procedimento descrito por Rosa et al. ${ }^{18}$ e liofilizadas em equipamento Savant Mod. E-C.

Fracionamento das substâncias húmicas aquáticas utilizandose ultrafiltração seqüencial em múltiplos estágios e fluxo tangencial - SFSUF

A amostra de SHA foi fracionada pelo SFSUF, desenvolvido por Rocha $e t a l^{16}$. Neste, a solução de SHA filtrada em membrana de porosidade $0,45 \mu \mathrm{m}\left(250 \mathrm{~mL}, 2,0 \mathrm{mg} \mathrm{mL}^{-1}, \mathrm{pH}=5,0\right)$ é bombeada em fluxo tangencial de $0,8-1,4 \mathrm{~mL} \mathrm{~min}^{-1} \mathrm{em}$ uma série de cinco filtros de membrana acoplados "in-line", levando à separação das seguintes frações por tamanho molecular: $\mathrm{F}_{1}>100 ; \mathrm{F}_{2} 100-50 ; \mathrm{F}_{3} 50-30 ; \mathrm{F}_{4}$ $30-10 ; \mathrm{F}_{5} 10-5 ; \mathrm{F}_{6}<5$ kDalton.

\section{Carbono orgânico dissolvido (COD)}

As determinações do COD nas frações $\left(\mathrm{F}_{1}-\mathrm{F}_{6}\right)$ foram feitas utilizando-se o método de combustão catalítica sob atmosfera de oxigênio em equipamento Shimadzu TOC-5000 Analyser.

\section{Análise elementar}

Determinou-se a composição elementar das frações em relação ao conteúdo de carbono, hidrogênio, nitrogênio e enxofre em analisador elementar CHNSO-CE Instruments, Perkin Elmer Mod. EA1110.

\section{Espectroscopia nas regiões do UV-VIS}

A partir de frações com mesmas massas de COD (50 mg), pH 5,0 e força iônica $0,1 \mathrm{~mol} \mathrm{~L}^{-1}$, obtiveram-se espectros UV/VIS por varreduras nos comprimentos de onda de 200-650 nm em espectrofotômetro de duplo feixe Hitachi U-2000. As razões $\mathrm{a}_{254} / \mathrm{a}_{436}$ foram determinadas pela razão entre os valores de absorbâncias em 254 e $426 \mathrm{~nm}^{19}$.

\section{Espectroscopia na região do infravermelho}

Os espectros na região do infravermelho das frações húmicas foram obtidos pela adição de $10 \mu \mathrm{L}$ de cada fração $(\mathrm{pH}$ 5,0) em 300 mg de $\mathrm{KBr}$ (grau espectroscópico), utilizando-se equipamento Nicolet modelo Nexus 670 equipado com acessório Smart Collector para análise por reflectância difusa.

\section{Determinação de metais por ICP-OES}

Cádmio, cobalto, crômio, cobre, ferro, manganês, níquel, chum- bo, zinco e alumínio ligados às frações $\left(\mathrm{F}_{1}-\mathrm{F}_{6}\right)$ foram determinados por espectrometria de emissão atômica por plasma de argônio induzido (Espectrômetro Thermo Jarrel Ash - IRIS-DUO), utilizando as respectivas linhas de emissão mais sensíveis e de acordo com as outras recomendações do fabricante e da literatura ${ }^{20}$.

Troca entre espécies metálicas originalmente complexadas em frações húmicas por íons $\mathrm{Cu}(\mathrm{II})$

Utilizou-se o sistema de ultrafiltração (Sartorius Ultrasart X), equipado com membrana de porosidade para tamanhos moleculares correspondentes a $1 \mathrm{kDa}$ e $47 \mathrm{~mm}$ de diâmetro (polyethersulfone, Gelman Pall-Filtron OMEGA), ilustrado na Figura 1. Alíquotas de cada fração (10-20 mL) foram diluídas a $200 \mathrm{~mL}$, o pH ajustado em 5,0 e adicionou-se solução $400 \mathrm{mg} \mathrm{L}^{-1} \mathrm{de} \mathrm{Cu}(\mathrm{II})$, até concentração final $40 \mathrm{mg} \mathrm{L}^{-1} \mathrm{em}$ cobre(II). Após intervalos de $10 \mathrm{~min}$, para estabelecimento do equilíbrio de troca entre as espécies metálicas originalmente complexadas pelas frações húmicas e íons $\mathrm{Cu}$ (II) adicionados, coletaram-se cerca de $2 \mathrm{~mL}$ do filtrado.

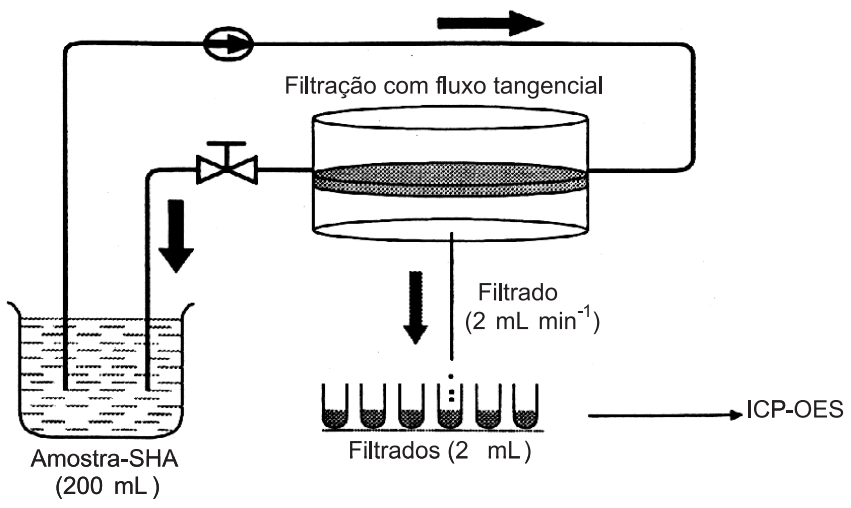

Figura 1. Esquema do procedimento analítico utilizado para estudo de troca entre espécies metálicas originalmente complexadas em frações húmicas aquáticas e íons Cu(II). Condições: sistema de ultrafiltração (Sartorius Ultrasart X), equipado com membrana de porosidade para tamanhos moleculares correspondentes a $1 \mathrm{kDa}$ e $47 \mathrm{~mm}$ de diâmetro (polyethersulfone, Gelman Pall-Filtron OMEGA)

\section{RESULTADOS E DISCUSSÃO}

\section{Fracionamento e caracterização das frações húmicas}

Rocha et al. ${ }^{16}$ desenvolveram um sistema de ultrafiltração seqüencial em múltiplos estágios e fluxo tangencial (SFSUF), para fracionamento de substâncias húmicas aquáticas (SHA) com base no tamanho molecular, utilizando-se ultrafiltração em filtros de membrana. Este sistema permite obter frações de SHA com diferentes tamanhos moleculares e, após o fracionamento, é possível caracterizar propriedades físico-químicas, tais como capacidade complexante com espécies metálicas, distribuição de grupos funcionais/estruturas reativas, estabilidades dos complexos M-SHA etc. Utilizando o SFSUF, fracionaram-se as SHA extraídas de amostras de água coletadas no Rio Itapanhaú-SP. Obtiveram-se e caracterizaram-se 6 frações húmicas com diferentes tamanhos moleculares $(>100-<5$ $\mathrm{kDa}$ ). Determinaram-se nas frações os teores de carbono orgânico dissolvido (COD), composição elementar (C, H, N, S), estimaramse a presença de grupos funcionais e condensação estrutural utilizando-se espectroscopias nas regiões do infravermelho e do UV-Vis, respectivamente. A Tabela 1 lista os resultados referentes a caracterizações das frações húmicas. 
Tabela 1. Resultados referentes a determinações de carbono orgânico dissolvido, composição elementar (\%), razões atômicas (H/C, C/N) e razão de absorbâncias em 254 e 436 nm de frações húmicas obtidas de amostras de água coletadas no Rio Itapanhaú-SP, utilizando-se o sistema de ultrafiltração sequencial em múltiplos estágios e fluxo tangencial

\begin{tabular}{lccccccc}
\hline Frações $(\mathrm{kDa})$ & COD mg L & $\% \mathrm{~N}$ & $\% \mathrm{C}$ & $\% \mathrm{H}$ & $\mathrm{H} / \mathrm{C}$ & $\mathrm{C} / \mathrm{N}$ & $\mathrm{a}_{254} / \mathrm{a}_{436}$ \\
\hline $\mathrm{F}_{1}(>100)$ & 1250,8 & 0,61 & 37,85 & 1,44 & 0,46 & 72,25 & 10,28 \\
$\mathrm{~F}_{2}(100-50)$ & 494,12 & 1,31 & 37,11 & 3,94 & 1,27 & 33,05 & 11,28 \\
$\mathrm{~F}_{3}(50-30)$ & 1725,76 & 1,29 & 37,19 & 3,92 & 1,26 & 33,63 & 10,54 \\
$\mathrm{~F}_{4}(30-10)$ & 1722,04 & 1,51 & 36,27 & 3,9 & 1,29 & 28,02 & 11,45 \\
$\mathrm{~F}_{5}(10-5)$ & 619,84 & 1,54 & 33,17 & 3,59 & 1,30 & 25,13 & 16,94 \\
$\mathrm{~F}_{6}(<5)$ & 40,91 & 3,33 & 23,12 & 0,59 & 1,00 & 8,13 & 19,74 \\
\hline
\end{tabular}

As razões atômicas $\mathrm{H} / \mathrm{C}$ e $\mathrm{C} / \mathrm{N}$ geralmente têm sido utilizadas para estimativa do grau de aromaticidade e humificação de substâncias húmicas, respectivamente. Excetuando-se $\mathrm{F}_{1}$, há similaridades entre os valores das razões molares $\mathrm{H} / \mathrm{C}$ das diferentes frações, os quais estão próximos aos valores disponíveis na literatura ${ }^{21}$ e indicam elevada aromaticidade. Dentre todas as frações, $\mathrm{F}_{1}$ e $\mathrm{F}_{6}$ apresentaram menores razões atômicas $\mathrm{H} / \mathrm{C}$ indicando tratarem-se das frações mais aromáticas. As razões atômicas $\mathrm{C} / \mathrm{N}$ das frações $\mathrm{F}_{2}, \mathrm{~F}_{3}, \mathrm{~F}_{4}$ e $\mathrm{F}_{5}$ mostram comportamento semelhante, indicando similaridades quanto ao grau de humificação. A fração $\mathrm{F}_{1}$ apresentou elevada razão C/N $(72,25)$ indicando tratar-se da fração mais humificada, enquanto a fração $\mathrm{F}_{6}$, de menor tamanho molecular, apresenta razão $\mathrm{C} /$ $\mathrm{N}$ cerca de 4 vezes menor, indicando menor grau de humificação desta em relação às demais frações. A baixa razão $\mathrm{C} / \mathrm{N}$ para $\mathrm{F}_{6}$ pode estar associada à presença de compostos menos humificados como, por exemplo, aminoácidos e proteínas ${ }^{21}$.

Devido à superposição de bandas, o espectro de absorção na região UV-Vis de ácidos húmicos e fúlvicos não tem bandas bem definidas, apresentando alguns máximos na região do ultravioleta ${ }^{21}$. De acordo com Abbt-Braun ${ }^{19}$, a razão entre as absorbâncias em 254 e $436 \mathrm{~nm}\left(\mathrm{a}_{254} / \mathrm{a}_{436}\right)$ é um parâmetro interessante para a caracterização de substâncias húmicas em solução, permitindo uma estimativa do grau de condensação. De acordo com a Tabela 1, verificaram-se que as frações $F_{1}, F_{2}, F_{3}$ e $F_{4}$ apresentaram razões de absorbâncias similares e menores que as frações $\mathrm{F}_{5}$ e $\mathrm{F}_{6}$. Estas, devido aos maiores valores, possuem características menos condensadas, sendo estas observações corroboradas pelos resultados das razões molares H/C.

A Figura 2 mostra os espectros na região do infravermelho para as frações húmicas de diferentes tamanhos moleculares.

Os espectros de infravermelho mostram bandas de absorção características de substancias húmicas aquáticas. As frações apresentaram bandas ao redor de $3400 \mathrm{~cm}^{-1}$ referentes ao estiramento $\mathrm{OH}$ de fenóis e/ou ácidos carboxílicos e/ou estiramento NH de aminas. Picos na região de $2900 \mathrm{~cm}^{-1}$ indicam a presença de estiramento $\mathrm{CH}$ de grupos alifáticos $\left(\mathrm{CH}_{2}\right.$ e $\left.\mathrm{CH}_{3}\right)$. As bandas nas regiões de 1630 e 1720 $\mathrm{cm}^{-1}$ são devidas a vibrações de carbonílas de grupos carboxilatos e/ ou carboxílicos e/ou cetonas. Picos em torno de $1385 \mathrm{~cm}^{-1}$ e bandas na região de $1035-1100 \mathrm{~cm}^{-1}$ estão associados ao estiramento do grupo carboxilato e estiramento $\mathrm{CO}$ de álcoois, respectivamente ${ }^{20,22}$. Comparando-se os espectros das frações de diferentes tamanhos moleculares, verificam-se praticamente as mesmas bandas de absorção, indicando grande similaridade entre os grupos funcionais presentes nas estruturas das frações.

\section{Distribuição de carbono orgânico dissolvido e metais}

A Figura 3 mostra a distribuição das frações de SHA em função das respectivas porcentagens de COD e de íons metálicos originalmente complexados. Observaram-se que as frações $\mathrm{F}_{3}(50-30 \mathrm{kDa})$ e $\mathrm{F}_{4}(30-10 \mathrm{kDa})$ apresentaram maiores teores, enquanto a fração $\mathrm{F}_{6}$

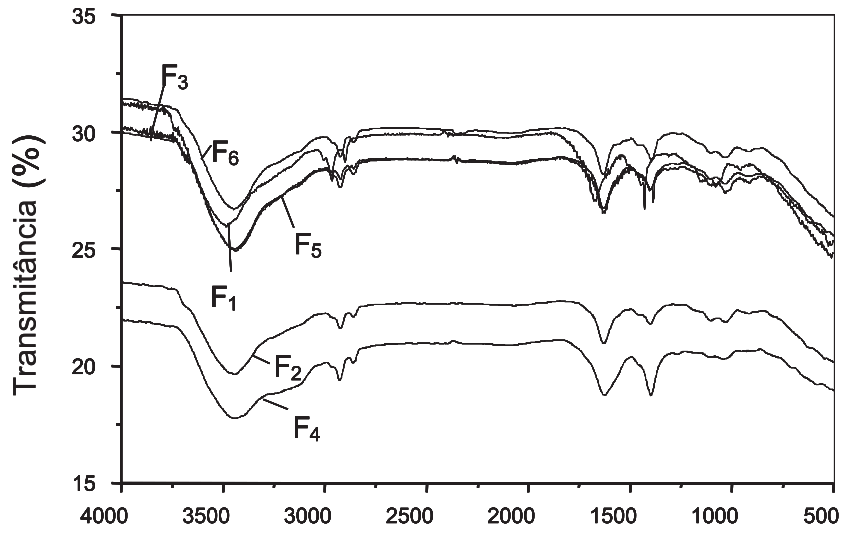

Número de onda $\left(\mathrm{cm}^{-1}\right)$

Figura 2. Espectros na região do infravermelho para as frações húmicas de diferentes tamanhos moleculares, extraídas de amostras de água coletadas no Rio Itapanhaú-SP, utilizando-se sistema de ultrafiltração sequencial em múltiplos estágios e fluxo tangencial. Condições: $250 \mathrm{~mL} S H A 2 \mathrm{mg} \mathrm{mL}^{-1}$; pH 5,0; frações $F_{1}:>100 ; F_{2}: 50-100 ; F_{3}: 30-50 ; F_{4}: 10-30 ; F_{5}: 5-10 ; F_{6}<5$ $k D a$

$(<5 \mathrm{kDa})$ mostrou menor teor de COD. A partir dos resultados obtidos, pode-se estabelecer a seguinte ordem decrescente de distribuição de carbono nas diferentes frações: $\mathrm{F}_{3}, \mathrm{~F}_{4}>\mathrm{F}_{1}>\mathrm{F}_{5}, \mathrm{~F}_{2}>>\mathrm{F}_{6}$.

Com relação aos íons metálicos, de maneira geral, as maiores porcentagens de espécies metálicas originalmente complexadas nas SHA estão nas frações com maiores porcentagens de COD, ou seja, $\mathrm{F}_{3}, \mathrm{~F}_{4}$ e $\mathrm{F}_{1}$. Assim, há relação direta entre concentração de metais complexados e COD, podendo-se estabelecer para os metais a mesma ordem de distribuição observada para o COD.

Sargentini et al. ${ }^{6}$ estudando frações húmicas de SHA extraídas do Rio Negro-AM verificaram menor quantidade de carbono orgânico na fração $F_{1}(>100 \mathrm{kDa})$ e a maior quantidade na fração $\mathrm{F}_{6}(<5$ $\mathrm{kDa})$. Também observaram que a maior porcentagem de íons metálicos originalmente complexados estava presente na fração $F_{2}$ (50-100 $\mathrm{kDa}$ ). Comparando os resultados obtidos por esses autores com os já discutidos neste trabalho, pode-se inferir que não há similaridade entre a capacidade e a afinidade de complexação entre frações húmicas aquáticas, extraídas de amostras de água coletada de diferentes localidades (Rio Negro-AM e Córrego Itapitangui-SP).

\section{Determinação de constantes de troca}

Para o estudo de troca entre espécies metálicas originalmente complexadas pelas frações húmicas e íons $\mathrm{Cu}$ (II), utilizou-se o procedimento analítico proposto por Burba et al. ${ }^{13}$ ilustrado na Figura 1. 


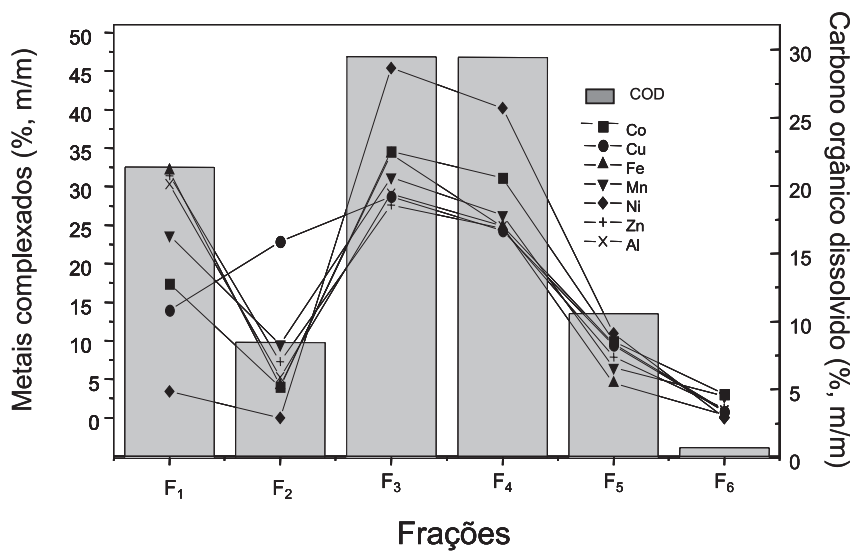

Figura 3. Distribuição de frações húmicas aquáticas extraídas de amostra de água coletada no Rio Itapanhaú localizado no município de Bertioga$S P$, em função de suas respectivas percentagens de carbono e de íons metálicos originalmente complexados. Condições: $250 \mathrm{~mL} \mathrm{SHA}, 2 \mathrm{mg} \mathrm{mL} \mathrm{L}^{-1}$, pH 5,0; frações $F_{1}:>100 F_{2}: 100-50, F_{3}: 50-30, F_{4}: 30-10, F_{5}: 10-5$ e $F_{6}<$ $5 \mathrm{kDa}$

O equilíbrio alcançado entre $\mathrm{M}-\mathrm{SHA}(\mathrm{M}=\mathrm{Fe}, \mathrm{Al}$ e $\mathrm{Co})$ e a troca pelos íons $\mathrm{Cu}(\mathrm{II})$ pode ser descrito pelas razões $\mathrm{M}_{\text {livre }}$ / M-SHA em função da razão crescente de ions $\mathrm{Cu}(\mathrm{II})_{\text {live }} / \mathrm{Cu}-\mathrm{SHA}$, como mostrado na Figura 4. Considerando que no estado de equilíbrio a lei de ação das massas é obedecida, de acordo com a equação (1), é possível determinar os valores das constantes de troca entre as espécies $\mathrm{Fe}, \mathrm{Al}$ e Co por íons $\mathrm{Cu}(\mathrm{II})$.

$\mathrm{M}-\mathrm{SHA}+\mathrm{Cu} \rightarrow \mathrm{Cu}-\mathrm{SHA}+\mathrm{M}$

$$
\mathrm{K}_{\text {troca }}=\frac{[\mathrm{Cu}-\mathrm{SHA}][\mathrm{M}]}{[\mathrm{M}-\mathrm{SHA}][\mathrm{Cu}]}
$$

(as cargas são omitidas para simplificação)

onde $[\mathrm{M}]$ e $[\mathrm{Cu}]$ são determinadas nos filtrados obtidos por ultrafiltração com o sistema de ultrafiltração ilustrado na Figura 1; $[\mathrm{M}-\mathrm{SHA}]=\mathrm{C}_{\text {metal total }}-[\mathrm{M}]$, onde $\mathrm{C}_{\text {metal total }}$ é a concentração de metal originalmente complexado pela SHA determinada no tempo zero, ou seja, a primeira alíquota do filtrado antes da adição de íons $\mathrm{Cu}$ (II); $[\mathrm{Cu}-\mathrm{SHA}]=\mathrm{Cu}_{\text {adicionado }}-[\mathrm{Cu}]$, onde $\mathrm{Cu}_{\text {adicionado }}$ é o somatório da concentração de íons $\mathrm{Cu}$ (II) adicionados para cada intervalo de tempo.

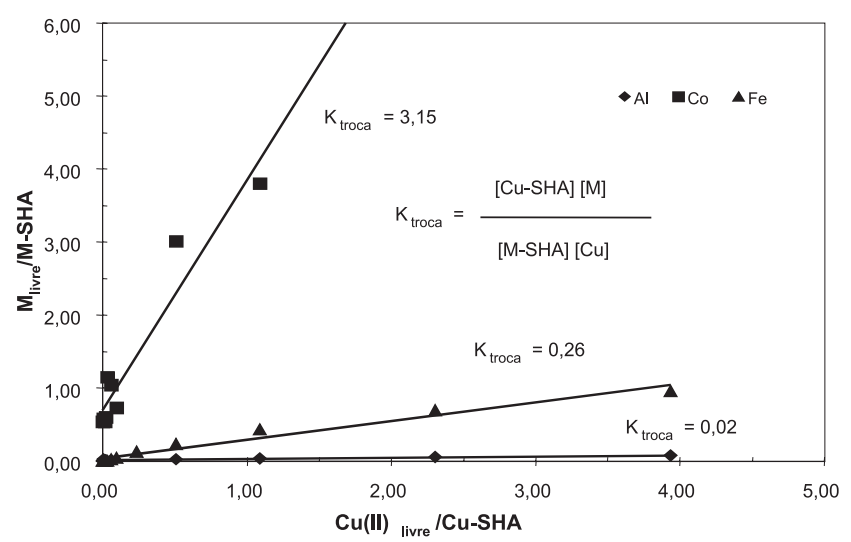

Figura 4. Equilíbrio de troca entre $M-S H A(M=C o, A l$ e Fe) e íons $\mathrm{Cu}(\mathrm{II})$ da fração $F_{3}(50-30 \mathrm{kDa})$
Na Tabela 2 estão expressas as constantes de troca determinadas para as várias frações húmicas, as quais caracterizam a troca entre espécies metálicas originalmente complexadas pelas SHA e íons $\mathrm{Cu}(\mathrm{II})$.

O procedimento está baseado em ultrafiltração utilizando-se membrana de $1 \mathrm{kDa}$. Entretanto, $\mathrm{F}_{6}(<5 \mathrm{kDa})$ pode conter espécies metálicas complexadas em moléculas de SHA menores que $1 \mathrm{kDa}$. A passagem destas pela membrana causa erros experimentais, impossibilitando o tratamento matemático para a determinação da constante da referida fração.

De acordo com a equação 1 , o valor da constante de troca é inversamente proporcional à estabilidade do complexo M-SHA. As constantes de troca entre íons $\mathrm{Cu}$ (II) e os íons $\mathrm{Al}$ e Fe foram menores que $1 \mathrm{em}$ todas as frações estudadas, com exceção de $\mathrm{F}_{1}$, indicando que os complexos Al-SHA e Fe-SHA possuem maior estabilidade que os complexos $\mathrm{Cu}$-SHA. Comparando-se a estabilidade desses dois complexos nas diferentes frações, observa-se que o complexo Fe-SHA possui, em média, estabilidade aproximadamente 7 vezes menor que o complexo Al-SHA. Para o complexo Co-SHA foram determinadas constantes de troca sempre maiores que 1 , indicando menor estabilidade que os complexos Cu-SHA. Também para os complexos (Co-SHA), observaram-se maior variação das constantes de troca nas diferentes frações que para os complexos Al-SHA e FeSHA. Pode-se inferir que para íons trivalentes há menor dependência da estabilidade do complexo em função do tamanho molecular. Os resultados das constantes de troca dos complexos, apresentados na Tabela 2, permitem estabelecer a seguinte ordem de estabilidade dos metais: $\mathrm{Al} \gg>\mathrm{Fe}>\mathrm{Cu}>>\mathrm{Co}$. Estes resultados são corroborados por Burba et al. ${ }^{13}$, quando da caracterização "on site" de águas naturais foram determinados valores de $\mathrm{K}_{\text {troca }}<<1$ para complexos de formados entre SHA e espécies metálicas trivalentes.

Tabela 2. Constantes de troca entre íons $\mathrm{Cu}$ (II) e espécies metálicas nas frações húmicas de diferentes tamanhos moleculares

\begin{tabular}{lccccr}
\hline & \multicolumn{5}{c}{ Frações / constantes de troca $\left(\mathrm{K}_{\text {troca }}\right)$} \\
\cline { 2 - 6 } Metais & $\mathrm{F}_{1}$ & $\mathrm{~F}_{2}$ & $\mathrm{~F}_{3}$ & $\mathrm{~F}_{4}$ & $\mathrm{~F}_{5}$ \\
\hline Co & 3,76 & 2,81 & 3,15 & 1,10 & 24,24 \\
$\mathrm{Al}$ & 0,09 & 0,11 & 0,02 & 0,04 & 0,07 \\
$\mathrm{Fe}$ & 1,24 & 0,47 & 0,26 & 0,29 & 0,40 \\
\hline
\end{tabular}

\section{CONCLUSÕES}

O fracionamento com base no tamanho molecular de SHA extraídas por resina XAD-8 de amostra de água coletada no Rio Itapanhaú no município de Bertioga-SP, mostrou uma relação direta entre as porcentagens de COD e de metais originalmente complexados nas diferentes frações. Pode-se estabelecer a seguinte ordem decrescente de distribuição de carbono e metais nas diferentes frações: $\mathrm{F}_{3}$, $\mathrm{F}_{4}>\mathrm{F}_{1}>\mathrm{F}_{5}, \mathrm{~F}_{2}>\mathrm{F}_{6}$.

As razões atômicas $\mathrm{H} / \mathrm{C}$ sugerem que as frações $\mathrm{F}_{1}$ e $\mathrm{F}_{6}$ possuem maior aromaticidade, e as razões atômicas $\mathrm{C} / \mathrm{N}$ não inferem diferenças significativas quanto ao grau de humificação entre as 6 frações. Dados espectroscópicos obtidos por UV-Vis mostraram razões entre as absorbâncias $\mathrm{a}_{254} / \mathrm{a}_{436}$ com maiores valores para $\mathrm{F}_{4} \mathrm{e} \mathrm{F}_{5}$, provavelmente por possuírem características menos condensadas. As bandas de absorção na região do infravermelho indicaram grande similaridade entre os grupos funcionais presentes nas estruturas das diferentes frações.

O procedimento analítico de ultrafiltração utilizado possibilitou determinar constantes de troca entre íons $\mathrm{Cu}(\mathrm{II})$ adicionados e as 
espécies metálicas originalmente complexadas nas frações húmicas. Os complexos Al-SHA e Fe-SHA possuem maior estabilidade que os complexos Co-SHA. Além disto, para as espécies trivalentes (Al e Fe) caracterizou-se menor dependência da estabilidade do complexo M-SHA em função do tamanho molecular. Este trabalho contribui para melhor entendimento das interações entre espécies metálicas e substâncias húmicas aquáticas.

\section{AGRADECIMENTOS}

Ao Conselho Nacional de Pesquisa e Desenvolvimento (CNPq), Fundação de Amparo a Pesquisa do Estado de São Paulo (FAPESP) e Conselho Nacional de Aperfeiçoamento de Pessoal de Nível Superior (CAPES), por bolsas e suporte financeiro. Ao Dr. P. Burba do Institut für Spektrochemie und Angewandte Spektroskopie (ISAS) pelas discussões.

\section{REFERÊNCIAS}

1. Burba, P.; Jakubowski, B.; Kuckuk, R.; Kullmer, K.; Heumann, K. G.; Fresenius' J. Anal. Chem. 2000, 368, 689.

2. Rocha, J. C.; Sargentini Jr, É.; Zara, L. F.; Rosa, A .H.; Santos, A.; Burba, P.; Talanta 2000, 53, 551.

3. Lead, J.R.; Hamilton-Taylor, J.; Davison, W.; Harper, M.; Geochim. Cosmochim. Acta 1999, 63, 1661.

4. Ziechmann, W.; Hubner, M.; Jonassen, K. E. N; Batsberg, W.; Nielsen, T.; Hahner, S.; Hansen, P. E.; Gundmundson, A. L. Em Humic SubstanceVersatile Components Of Plants, Soils and Water; Ghabbour, E. A.; Davies, G., eds.; Royal Society of Chemistry: Boston, 2000, p. 9.

5. Piccolo, A.; Nardi, S.; Concheri, G.; Chemosphere 1996, 33, 602.
6. Sargentini Jr., E.; Rocha, J. C.; Rosa, A. H.; Zara, L. F.; Santos, A.; Quim Nova 2001, 24, 339.

7. Bubert, H.; Lambert, J.; Burba, P.; Fresenius' J. Anal. Chem. 2000, 368, 274.

8. Aster, B.; Von Bohlen, A.; Burba, P.; Spectrochim. Acta, Part B 1997, 52, 1009.

9. Shkinev, V. M.; Fedorova, O. M.; Spivakov, B.; Mattusch, J.; Wennrich, R.; Lohse, M.; Anal. Chim. Acta 1996, 327, 167.

10. Mccarthy, J. F. Em Aquatic Humic Substances/Influence on Fate and Treatment of Pollutants; Suffet, I. H.; MacCarthy, P., eds.; American Chemical Society: Washington, 1989, p. 263.

11. Ninfanteva, T. J.; Shkinev, V. M.; Spivakov, B. Ya.; Talanta 1999, 48, 257.

12. Burba, P.; Analytiker Taschenbuch, Springer: Berlin, 1998, p. 45.

13. Burba, P.; Van den Berg, J.; Klockow, D.; Fresenius' J. Anal. Chem., no prelo.

14. Burba, P.; Rocha, J. C.; Klockow, D.; Fresenius' J. Anal. Chem. 1994, 349 , 800.

15. Rocha, J. C.; Sargentini Jr., E.; Toscano, I. A. S.; Rosa, A. H.; Burba, P.; J. Braz. Chem. Soc. 1999, 10, 169.

16. Rocha, J. C.; Zara, L. F.; Rosa, A. H.; Sargentini Jr., E. ; Burba, P.; Quim. Nova 2000, 23, 410.

17. Malcolm, R.; Thurmann, E. M. Em Aquatic and Terrestrial Humic Materials; Christman, R. F.; Gjessing, E. T., eds.; Arbor Science, 1995, p.1.

18. Rosa, A. H.; Rocha, J. C.; Furlan, M.; Quim. Nova 2000, 23 , 472.

19. Abbt-Braun, G. Em Progress in Hydrogeochemistry; Matthess, G.; Frimmel, F. H., eds.; Springer: Berlin, 1992, p. 29.

20. Boss, C. B.; Fredeen, K. J.; Concepts, Instrumentation and Techniques in Inductively Coupled Plasma Optical Emission Spectrometry; Perkin Elmer, $2^{\text {a }}$ ed., 1997.

21. Stevenson, F. J.; Humus Chemistry: Genesis, Composition, Reactions, $2^{\text {a }}$ ed., John Wiley \& Sons INC: New York, 1994, p. 303.

22. Burba, P.; Shkinev, V.; Spivakov, B. Ya.; Fresenius'J. Anal. Chem. 1995, 351, 74. 